\title{
Do corticosteroids really alter mood?
}

\author{
D. M. MITCHELL \\ M.D., M.R.C.P. \\ J. V. COLLINS \\ M.D., F.R.C.P. \\ University College Hospital, London WCI and Brompton Hospital, London SW3
}

Corticosteroids are generally believed to induce a wide range of psychological changes. Textbooks of therapeutics, psychiatry and endocrinology record that administration of corticosteroid drugs or adrenocorticotrophin most commonly result in a high incidence of mood elevation, cheerfulness and optimism. Less frequently there is frank euphoria, irritability, insomnia, increased motor activity and hypomania. A smaller percentage of patients, particularly those on high doses of corticosteroids, become anxious or depressed and apathetic, while a still smaller percentage develop psychoses with hallucinations, delusions and features of depersonalization (Goodman and Gillman, 1980; Kolb and Brodie, 1982; Kaplan, Freedman and Sadlock, 1980; Reichlin, 1981).

These beliefs are based on case reports which started to appear shortly after the introduction of corticosteroids as therapeutic agents. Many report psychotic reactions after starting corticosteroid treatment (Borman and Schmallenberg, 1951; Clark, Quarton and Cobb, 1953). Euphoric and depressive responses are also described (Rome and Braceland, 1952), and such reports have continued to appear (Baloch, 1974; Sharma and Gajwani, 1973; Kauffman et al., 1982). However, evidence that corticosteroids are directly linked to psychological change based on such case reports is insufficient. A causal role for corticosteroids is suggested if the mental disturbance appears shortly after starting the drug and remits on stopping. Unfortunately such case reports are invariably complicated by the use of psychotropic drugs to control symptoms. Furthermore, most reports have been of the steroid-induced psychoses which are relatively easy to recognize and characterize. Even if a causal connection is established here it does not apply to the much commoner and more subtle changes in mood which are said to occur but which are much more difficult to quantitate.

An early study (Rome and Braceland, 1952) stressed the variety of psychological abnormalities seen and divided corticosteroid-induced mental changes into mood elevation, euphoria, psychoneu- rosis and psychosis. Five patients were described who were thought to have such steroid-induced changes -all of whom had had previous major psychiatric abnormalities. However, a causal link between introduction of steroids and worsening of psychological state is far from clear in this study, frequently quoted in subsequent literature, as insufficient detail is provided. Lewis and Fleminger (1954) tested the hypothesis that a previous psychiatric history was a necessary condition for the development of a steroid psychosis when treating 12 patients (eleven with rheumatoid arthritis and one with systemic lupus erythematosus) with an unequivocal history of mental disturbance with cortisone (maximum dose 100 $\mathrm{mg} /$ day), for concommitant organic disease. Patients were observed and underwent psychological testing for 6 weeks, received placebo for 10 days, and then received cortisone for 5.5-7.5 weeks. Cortisone was then withdrawn. All improved as regards symptoms of arthritis initially, and all except one relapsed on cortisone withdrawal. All had mental improvement in parallel with physical improvement. As pain and disability subsided, gloom and depression lifted, and returned when relapse occurred. The authors of this detailed study concluded that mood change was generally the result of symptom relief and that corticosteroids had no direct euphoriant effect. They also suggested that psychiatric history was not a contra-indication to steroid therapy.

Subsequently, larger studies have investigated various aspects of possible corticosteroid-induced mental change and give some indication of the frequency of such changes. In 240 in-patients on corticosteroids a correlation between dose, serum albumin and incidence of side effects was found. Ten patients $(2 \cdot 4 \%)$ developed psychoses (Lewis et al., 1971). In another report of 718 consecutive inpatients on cortico-steroids, $21(3 \%)$ patients developed acute psychological changes (Boston Collaborative Drug Surveillance Program, 1972). None of these patients were thought to be emotionally unstable before corticosteroid treatment. Eight had inappropriate euphoria, and 13 became psychotic, of whom 
two were depressed and six were maniacal. All remitted when corticosteroids were stopped or reduced although psychotherapeutic drugs were used in the 13 psychotic patients. The mean dose of prednisolone in patients with psychological side effects was $59 \mathrm{mg} /$ day whereas that of the whole group studied was $32 \mathrm{mg} /$ day. There was a significant relation between dose and incidence of mental change. Of 463 patients on less than $40 \mathrm{mg}$ prednisolone/day, the incidence was $1.3 \%$; of 175 patients on 41-80 mg/day, the incidence was $4.6 \%$ and of 38 patients on greater than $80 \mathrm{mg}, 18.4 \%$. However, it could be said that patients requiring very high doses of corticosteroids obviously have serious organic disease which itself carries a high incidence of psychiatric complications when compared with mild or moderate disease and perhaps the incidence of psychosis is surprisingly low in the patients requiring such high doses. This point is developed by Kauffman et al. (1982) who emphasize the difficulty of distinguishing between toxic psychosis induced by steroid drugs, delirium associated with severe organic disease, the direct effect of organic disease on the central nervous system from metastatic neoplasm, vasculitis, hypoxia or electrolyte disturbance, and coincidental functional psychosis. The incidence of psychosis in the Boston study (Boston Collaborative Drug Surveillance Program, 1972) agrees with that of Hall et al. (1979) who found that 5\% of patients taking more than $40 \mathrm{mg}$ prednisolone/day became psychotic between 2 and 23 days (mean 6 days) after starting treatment.

Further attempts to establish a causal relationship between corticosteroids and psychological disturbance have been made by making retrospective surveys of steroid-treated patients and comparing the incidence of psychosis with that in a control group of similar patients not on steroids. Clearly this approach is problematical as presumably there are good reasons why the study group is on steroids, and the control group is not. The most likely of these is that the steroid-treated group has more severe disease which carries a higher incidence of psychological complications anyway. Marx and Barker (1967) found that six of $50(12 \%)$ patients with ulcerative colitis developed acute psychotic reactions while on steroids whereas only two of $80(2.6 \%)$ patients not taking steroids developed psychoses. Such small differences barely reach statistical significance and do not support the general conclusion that steroid therapy can induce psychoses, particularly as the control group was not matched for disease severity, age or sex. Smyllie and Connolly (1968) compared the incidence of serious psychosis between two groups of patients with respiratory disease who were matched for age, sex, disease and psychiatric history. Ten of $550(1.8 \%)$ patients treated with cortico- steroids developed psychosis whereas 16 of 499 (3.2\%) patients not treated with corticosteroids developed psychosis. The authors of this study which involved comparatively large numbers of patients conclude that the risk of psychosis was not increased by steroids. However the majority of their patients were on the equivalent of $20 \mathrm{mg}$ or less of prednisolone/day.

Cordess, Felstein and Drachman (1981) studied the mental state of 72 patients with neuromuscular disorders, 39 of whom were on alternate day prednisolone (mean daily dose $35 \mathrm{mg} ; 65 \mathrm{mg}$ and $5 \mathrm{mg}$ on alternate days). They measured mental state using the General Health Questionnaire. The corticosteroid treated group were less anxious and depressed than the 33 non-steroid treated disease controls and the authors suggested that this might be due to a euphoriant action of the drug, although the control group not on steroids were said to be well matched with the steroid treated group. It is unclear why they were not also on treatment. If they were well matched, but not on steroids they probably had more symptoms and disability resulting in a higher incidence of psychological complaint.

In a prospective randomized study, Cade, Spooner and Schlein (1973) showed that nine of 28 (32\%) patients with lupus nephritis taking corticosteroids developed psychoses whereas none of $27(0 \%)$ patients not on steroids did so. However, patients with SLE have a high incidence of psychosis anyway (Stern and Robbins, 1960) and the steroid treated patients in this study received high doses of 60-100 $\mathrm{mg}$ prednisolone/day for 6 months. The Boston Collaborative Study (1972), as mentioned above, suggested an increased risk of psychosis for patients on very high doses of corticosteroids.

From these studies several conclusions can be drawn. Firstly there may be an increased risk of psychosis when high doses of corticosteroids are used (Cade et al., 1973; Boston Collaborative Drug Surveillance Program, 1972). Secondly, there is no firm evidence of increased risk when lower doses are used. Thirdly, these studies do not provide information regarding less florid psychological changes and their relation to corticosteroid therapy.

Further evidence for a link between psychological abnormalities and corticosteroids is often cited by considering Cushing's syndrome in which a high incidence of neuroses and psychoses is found (Trethowan and Cobb, 1952). Between $20 \%$ and $40 \%$ have mental disturbances, depression being the most common (Michael and Gibbons, 1963). Although psychological changes may precede physical change, reactive depression due to change in physical appearance also occurs (Kaplan et al., 1980). Less commonly behavioural disturbances occur with irritability, insomnia, loss of libido, anxiety, emotional 
lability, elated mood and hypomania. Rarely toxic confusional states or psychoses with delusions and hallucinations are observed. Psychological abnormalities in Cushing's disease are said to remit when the condition is treated (Reichlin, 1981).

However, a wide range of psychological change is also seen in Addison's disease, depression, fatigue and apathy being most common. Irritability and psychoses with delusions and hallucinations occur more rarely. All abnormalities are said to resolve following cortisol administration (Goodman and Gillman, 1980; Kaplan et al., 1980). It seems that both deficiency and excess of endogenous glucocorticoids result in similarly wide ranges of psychological disorder.

Links between elevated cortisol secretion rates and depressive illness have been suggested (Sclare and Grant, 1969). Gibbons (1962) found high cortisol secretion rates in 15 depressed patients, which fell in 14 on recovery. Cortisol levels rise in stress (Michael and Gibbons, 1963), and episodes of grief, anxiety and the stress of hospitalization for depressive illness may produce elevated cortisol levels which have nothing to do with depression per se. Long standing stable depressive illness alone does not seem to have major effects on cortisol levels (Sachar, 1967).

Serious organic diseases frequently have a high incidence of psychological disturbance either due to direct involvement of the central nervous system or as a result of chronic disability (Rome and Braceland, 1952). For example, there is a high incidence of psychosis in patients with systemic lupus erythematosis (Stern and Robbins, 1960), and this high incidence may be exacerbated by high dose corticosteroid therapy (Sergent et al., 1975). Generally corticosteroids used in treatment of this condition are not thought to play a part in mental disturbance (Heine, 1969).

It was initially thought that there might be a genetic predisposition to develop steroid psychoses (Rome and Braceland, 1952) and that a psychiatric history was a contraindication to treatment with corticosteroids (Copeman et al., 1954). It could be suggested that the low incidence of psychoses found in the Boston Collaborative Drug Surveillance Program (1972) is attributable to none of the patients studied having a psychiatric history. However, the prospective study of Lewis and Fleminger (1954) found that 12 patients with psychiatric history did not develop mental disturbance on cortisone. Although only a small number of patients were involved in this detailed study, one can conclude that psychiatric history does not invariably predispose to steroidinduced psychosis, and so psychiatric history is not a contraindication to treatment.

If there is little evidence to support the view that corticosteroids produce mental changes, there is even less regarding their mode of action. The most likely explanation is that feelings of well being and euphoria occur when corticosteroid treatment alleviates symptoms (Goodman and Gillman, 1980; Lewis and Fleminger, 1954). Alternatively psychological disturbance due to direct central nervous system involvement by the disease process from, say, vasculitis, hypoxia, or electrolyte disturbance may resolve following effective steroid-induced control of the disease. Many other mechanisms for steroid-induced psychological change have been suggested including direct damage to neural tissue or related vasculature, fluid shifts due to electrolyte changes, biochemical and enzyme changes in neurones and a direct neuropharmacological effect of the drug producing euphoria (Quarton et al., 1955). There is no convincing evidence for any of these mechanisms which must therefore remain purely speculative.

A different issue that requires separate consideration is the question of mental changes which may occur on stopping corticosteroids. Some patients have been reported to become psychologically dependent on corticosteroids and to experience a variety of mental disturbances on their withdrawal including psychotic reactions, depression and agitation (Kimball, 1971; Morgan, Boulnois and BurnsCox, 1973).

\section{Conclusion}

This brief survey shows that a very wide range of mental disturbances has been attributed to corticosteroids acting by many possible mechanisms, but that the evidence that they really cause psychological change is thin and largely circumstantial. Actual case series are often vague regarding details of criteria for inclusion and methods, if any, of psychological measurement. It can be very difficult to distinguish effects of disease from effects of treatment. Remission of the mental disorder on stopping corticosteroids is suggestive of a causal link, but this does not exclude the possibility of disease-induced psychosis for if it is possible to reduce steroids on the basis of mental disturbance, presumably the underlying disease has improved. Even in cases where psychosis has resolved on stopping corticosteroids, the situation is complicated by the inevitable use of psychotropic drugs to control symptoms. However, the available evidence suggests that some cases of mental disturbance may indeed result from corticosteroid therapy particularly if a high dosage is used. A wide range of changes have been reported, psychosis, euphoria and depression being the commonest. Perhaps this extreme variability in clinical picture argues against a straightforward causal relationship. A psychiatric history does not seem to predispose to such mental changes. 
It is clearly important to know if corticosteroids are genuinely euphoriant as, if they are, part of their beneficial effect in the chronic conditions for which they are used may be due to this rather than to a direct action on the disease process itself. Although corticosteroids have been in use clinically for over a quarter of a century their precise mode of action in the patient as a whole remains unclear although there is much information regarding their effects at a cellular level. There is a need for detailed controlled prospective studies of the effect of corticosteroids on psychological status, in a variety of conditions where they remain standard treatment.

\section{References}

BAloch, N. (1974) Steroid psychosis: A case report. British Journal of Psychiatry, 124, 545.

Boston Collaborative Drug Surveillance Program (1972) Acute adverse reactions to prednisolone in relation to dosage. Clinical Pharmacology and Therapeutics, 13, 694.

Borman, M.C. \& SCHMALlenberg, H.C. (1951) Suicide following cortisone treatment. Journal of the American Medical Association. 146, 337.

Cade, R., Spooner, G. \& Schlein, E. (1973) Comparison of azathioprine, prednisone and heparin alone or combined in treating lupus nephritis. Nephron, 10, 37.

Clark, L.D., Quarton, G.C., Cobb, S. \& Bauer, W. (1953) Further observations on mental disturbances associated with cortisone and ACTH therapy. New England Journal of Medicine, 249, 178.

Copeman, W.C.S., Dodds, C., Savage, O., Glyn, J.H. \& FEARNLEY, M.E. (1954) Management of rheumatoid arthritis with prolonged cortisone administration. British Medical Journal, 1, 1109.

Cordess, C., Felstein, M. \& Drachman, D. (1981) Psychiatric effects of alternate day steroid therapy. British Journal of Psychiatry, 138, 504.

GiBBONS, J.L. (1962) Cortisol secretion in depressive illness. Archives of General Psychiatry, 10, 572.

Goodman, L.S. \& Gillman, A. (1980) The Pharmacological basis of therapeutics. 6th edn., p. 1470. MacMillan, New York.

Hall, R., Popkin, M., Stickney, S. \& Gardner, E. (1979) Presentation of the steroid psychoses. Journal of Nervous and Mental Diseases, 167, 229.

HEINE, B.E. (1969) Psychiatric aspects of systemic lupus erythematosis. Acta Psychiatria Scandinavia, 45, 307.

KaPlan, H.I., Freeman, A.M. \& Sadlock, B.J. (1980) Comprehensive Textbook of Psychiatry. 3rd edn., p. 331. Williams and Wilkins, Baltimore, London.
Kauffman, M., Kahaner, K., Peselow, E.D. \& Gerson, S. (1982) Steroid psychoses: Case report and brief overview. Journal of Clinical Psychiatry, 43, 75.

KIMBaLl, C.P. (1971) Psychological dependency on steroids? Annals of Internal Medicine, 75, 111.

KOLB, L.C. \& BrodIE, H.K.H. (1982) Modern Clinical Psychiatry. 10th edn., p. 328. W. B. Saunders, Philadelphia.

LewIS, A. \& Fleminger, J.J. (1954) The psychiatric risk from corticotrophin and cortisone. Lancet, i, 383.

LeWIS, G.P., Jusko, W.J., BURKE, C.W. \& Graves, L. (1971) Boston Collaborative Drug Surveillance Program. Prednisolone side effects and serum protein levels. A collaborative study. Lancet, ii, 778.

MARX, F.W. \& BARKER, W.F. (1967) Surgical results in patients with ulcerative colitis treated with and without corticosteroids. American Journal of Surgery, 113, 157.

MiChAEL, R.P. \& GiBBONS, J.L. (1963) Inter-relationships between the endocrine system and neuro-psychiatry. International Review of Neurobiology, 5, 243.

MORGAN, H.E., BoulnoIs, J. \& BuRns-Cox, C. (1973) Addiction to prednisone. British Medical Journal, 2, 93.

Quarton, G.C., Clark, L.D., Cobb, S. \& Bauer, W. (1955) Mental disturbances associated with ACTH and cortisone. A review of explanatory hypotheses. Medicine, 34, 13.

ReICHLIN, S. (1981) In: Textbook of Endocrinology, (Ed. R.H. O Williams). 6th edn. W. B. Saunders, Philadelphia and London, p. 820.

RoME, H.P. \& BRACEland, F.J. (1952) Psychological responses to ACTH, cortisone and related steroid substances, Journal of the American Medical Association, 148, 27.

SACHAR, E.J. (1967) Corticosteroids in depressive illness. Archives of General Psychiatry, 17, 544.

SClARE, A.B. \& GRANT, J.K. (1969) Plasma 11-hydroxycorticosteroid concentrations in depressive illness. Journal of Endocrinology, 43, 677.

SERGent, J.S., LockShin, M.D., KLEMPNER, M.S. \& LiPSKY, B.A. (1975) Central nervous system disease in systemic lupus erythematosis. American Journal of Medicine, 58, 644.

Sharma, S. \& GAJWANI, A.K. (1973) Prednisolone induced depression. Journal of the Association of Physicians of India, 21, 909.

SMYLLie, H.C. \& ConNolly, C.K. (1968) Incidence of serious complications of corticosteroid therapy in respiratory disease. Thorax, 23, 571.

Stern, M. \& Robbins, E.S. (1960) Psychoses in systemic lupus erythematosis. Archives of General Psychiatry, 3, 205.

TRETHOWAN, W.H. \& COBB, S. (1952) Neuropsychiatric aspects of Cushing's syndrome. Archives of Neurology and Psychiatry, 67, 283.

(Accepted 1 February 1984) 